\title{
Double-lobectomy in a steatotic liver transplantation rat model
}

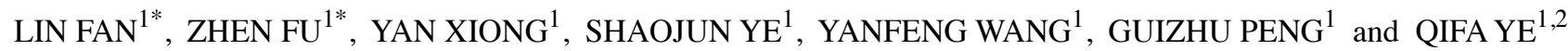 \\ ${ }^{1}$ Zhongnan Hospital of Wuhan University, Institute of Hepatobiliary Diseases of Wuhan University, \\ Transplant Center of Wuhan University, Hubei Key Laboratory of Medical Technology on Transplantation, \\ Wuhan, Hubei $430071 ;{ }^{2}$ The 3rd Xiangya Hospital of Central South University, \\ Research Center of National Health Ministry on Transplantation Medicine Engineering and Technology, \\ Changsha, Hunan 410013, P.R. China
}

Received March 28, 2020; Accepted September 16, 2020

DOI: $10.3892 / \mathrm{etm} .2021 .9687$

\begin{abstract}
Establishing a steatotic liver transplantation animal model can be a challenging process, which requires complex microsurgical technologies. The present study established a novel rat model of stable steatotic liver transplantation for marginal liver graft research, which notably minimized the number of animals used for the experiment. Briefly, male Sprague-Dawley rats $(\mathrm{n}=90)$ were fed with a high-fat diet (HFD; 60\%, kJ) or standard chow diet (SCD) for 8 weeks. The liver enzymes and lipid levels were assessed every week, and the degree of steatosis was determined via hematoxylin and eosin and Oil Red O staining. The results demonstrated that there were no significant differences in alanine aminotransaminase and aspartate aminotransferase levels between the SCD and HFD groups $(\mathrm{P}>0.05)$, whereas the level of plasma triglyceride (TG) increased by 1.76 -fold in the HFD group at week 2 , and progressively decreased to baseline levels by week 8 . Significantly higher levels of TG were observed in the HFD group compared with the SCD group at week 2 $(\mathrm{P}<0.05)$. In addition, the levels of plasma glucose $(\mathrm{P}<0.05)$, portal insulin $(\mathrm{P}<0.05)$ and content of liver lipid $(\mathrm{P}<0.01)$ increased in the HFD group compared with the SCD group. After 6 weeks, the liver steatosis was successfully formed and stable. Consequently, a rat liver developed hepatic macrovesicular steatosis $>60 \%$, which was subsequently used for transplantation after double-lobectomy. Post-transplantation survival rates in the HFD and SCD groups were as follows:
\end{abstract}

Correspondence to: Dr Qifa Ye, Zhongnan Hospital of Wuhan University, Institute of Hepatobiliary Diseases of Wuhan University, Transplant Center of Wuhan University, Hubei Key Laboratory of Medical Technology on Transplantation, 169 East Lake Road, Wuhan, Hubei 430071, P.R. China

E-mail: yqf_china@163.com

*Contributed equally

Key words: liver transplantation, hepatic steatosis, reduced size, animal model
Week 1,80 vs. $100 \%$ and 1 month, 20 vs. $100 \%$. A total of 20 rats were not sacrificed by performing double-lobectomy for biopsy. Taken together, the results of the present study suggest that rat liver double-lobectomy may be safely applied in steatotic liver transplantation without the need to sacrifice a large number of animals.

\section{Introduction}

Liver transplantation (LTx) is the most effective treatment for patients with end-stage liver diseases, including hepatic cirrhosis, hepatocellular carcinoma, hepatic hemangioma and hepatic echinococcosis, which has been extensively applied in the clinical setting for more than half a century (1-5). The discrepancy between donor organ supply and demand continues to increase with the growing number of potential candidates on the transplant waiting list (6). Thus, a series of strategies have been advocated to expand the donor pool from the extended criteria donors (ECD), including donation after cardiac death, age-related mortality, and malignancy and hepatic steatosis (7-9). However, liver grafts donated from ECD are associated with a higher risk of primary non-function (PNF), early allograft dysfunction and mortality (10).

Steatotic liver, which belongs to marginal liver grafts (MLG), is the most common liver disorder in developed countries, with a prevalence of $20-30 \%$ among the general population (11-16). Hepatic steatosis is typically characterized by the accumulation of lipid droplets in hepatocytes and is commonly divided into two subtypes, macrovescicular steatosis or microvescicular steatosis, based on the size of the fat vacuole and the location of the nucleus (17). Macrovesicular steatosis is defined as the accumulation of large fat droplets in the hepatocyte, displacing the nucleus to the edge of the cell, whereas microvesicular steatosis is defined as the presence of tiny lipid vesicles in the hepatocyte, without nuclear displacement (17). In the clinical setting, steatosis has been classified into mild $(<30 \%)$, moderate $(30-60 \%)$ or severe $(>60 \%)$ depending on the degree of the fatty infiltration (18).

Hepatic steatosis, particularly that in the liver with $30 \%$ macrosteatosis, is considered an independent risk factor for PNF due to increased susceptibility to ischemia-reperfusion injury (17,19-21). The following mechanisms have been 
proposed for hepatic steatosis: Liver microcirculation is hampered with excessive fat accumulation, which leads to mitochondrial damage, and oxidative stress during reperfusion, coupled with inflammatory response involving lipid peroxidation and leukocyte adhesion may contribute to the graft failure following transplantation $(20,22,23)$. Ploeg et al (18) reported that PNF rates increase up to $80 \%$ in the severely steatotic liver. In addition, previous studies have demonstrated that severely steatotic graft is associated with high PNF rates $(0-66 \%)$ and a 1-year graft with a survival rate of $25-90 \%(18,24-26)$. Currently, macrovesicular steatosis of $>30 \%$ is considered an independent predictor for a reduction of PNF and 1-year graft survival (27). Thus, the evaluation and restoring of organs from ECD attract increasing attention.

Due to difficulties and complexities in microsurgical technology (28), establishing a rat steatotic liver transplantation model can be challenging. In addition, donor livers or grafts require multiple biopsies at the point of procurement, during preservation and after transplantation. When using liver transplantation models, a single biopsy requires animals to be sacrificed, resulting in an increased number of experimental animals, which is not conducive to animal welfare and ethical principles. Thus, the present study established a novel method to decrease the number of experimental animals. The results of the present study suggest that liver tissues from different parts at different time points may significantly decrease the number of experimental animals used. In addition, the proposed method did not affect post-operative animal mortality (papillary process and quadrate lope excision: Partial liver transplantation vs. whole liver transplantation survival; $5 / 5$ vs. $5 / 5$ ).

The steatotic liver animal model induced by a high-fat diet (HFD) is commonly applied to assess hepatic steatosis in vivo. This model can mimic the etiology of hepatic steatosis in human beings (29). The present study aimed to establish a stable and reproducible steatotic liver model induced by a HFD that may be used to investigate the efficacy of reduced size transplantation in MLG research.

\section{Materials and methods}

Animals. A total of 90 male Sprague-Dawley rats $(210 \pm 10 \mathrm{~g})$, aged 6-8 weeks, were obtained from Hubei Provincial Center for Disease Control and Prevention in China. All animals were housed in an environment with a temperature of $23 \pm 1^{\circ} \mathrm{C}$, relative humidity of $55 \pm 10 \%$, air exchange $12-14$ times $/ \mathrm{h}$, a light/dark cycle of $12 / 12 \mathrm{~h}$, and were provided with food and tap water ad libitum. The present study was approved (IRB approval no. AF-177) by Wuhan University Institutional Animal Care and Use Committee (Wuhan, China).

A total of 10 rats were procured after 7 days of acclimation to provide baseline values. A total of five rats in the HFD group or standard chow diet (SCD) group were samples each week during 8 weeks of modeling. Rats were randomly divided into 8 groups $(n=10)$. Body weight and food intake were monitored on a daily basis. The time schedules for blood extraction in relation to the frozen sections used for analysis are presented in Fig. 1.

Dietary interventions. The HFD consisted of $60 \%$ lipid, $20.6 \%$ carbohydrate and $19.4 \%$ protein $(\mathrm{kJ})$, and was provided in rods direct from the manufacturer (Trophic Animal Feed
High-Tech Co., Ltd.). The lipids included in the HFD consisted of 90\% lard and 10\% soybean oil (Trophic Animal Feed High-Tech Co., Ltd.). The SCD consisted of usual pellet rat chow (Trophic Animal Feed High-Tech Co., Ltd.). In order to avoid fatty diarrhea, rats in the HFD group were fed using the following 6 day schedule: i) 2 days of $30 \%$ weight (wt) HFD and $70 \%$ wt SCD; ii) 2 days of 50\% wt HFD and 50\% wt SCD, and iii) 2 days of $70 \%$ wt HFD and $30 \%$ wt SCD.

Graft procurement and reduced size procedure. Anesthesia during liver procurement and transplantation was maintained using isoflurane (cat. no. R510-22-4; RWD Life Technology Co., Ltd.; $4 \%$ isoflurane for induction and $2 \%$ isoflurane for maintenance). Briefly, heparin (100 IU) in $2 \mathrm{ml}$ saline solution (cat.no.H8060-1g; Beijing Solarbio Science \& Technology Co., Ltd.) was injected into the penile vein, and a $5 \mathrm{~mm}$ long stent prepared from polyethylene tube was inserted into the common bile duct (CBD) and secured with 6-0 sutures. Livers were flushed in situ with $20 \mathrm{ml}$ of University of Wisconsin (UW) solution at $4^{\circ} \mathrm{C}$. A total of two hepatic lobes, the papillary process and the quadrate lobe were procured for assessment, using the liver volume reduction method illustrated in Fig. 2. The papillary process (Fig. 2C) was removed to assess the extent of hepatic steatosis before cold storage (CS) at $4^{\circ} \mathrm{C}$. The quadrate lobe (Fig. 2D) was cut following preservation with UW solution at $2-4^{\circ} \mathrm{C}$ for $4 \mathrm{~h}$. Venous cuffs prepared from two sizes of polyethylene tubes were subsequently placed in the portal vein (PV) and intrahepatic inferior vena cava (IHVC). The inside/outside diameters of polyethylene tube for CBD, PV and IHVC were 0.6/1.0, 1.8/2.2 and 2.8/3.2 mm, respectively. Grafts were stored in UW solution at $4^{\circ} \mathrm{C}$ for $4 \mathrm{~h}$ prior to implantation (Fig. 2E).

Serum analyses. Blood was drawn from the rats every week during the modeling process to detect hepatocyte injury and serum lipid levels via alanine aminotransaminase (ALT), aspartate aminotransferase (AST), triglyceride (TG), total cholesterol (TC), free fatty acid, high-density lipoprotein and low-density lipoprotein. These indices were measured at the Institute for Clinical Biochemistry and Diagnostics, Zhongnan Hospital of Wuhan University (Wuhan, China). Lipids from rat livers were prepared using chloroform-methanol extraction (30). Plasma glucose concentration was detected using a glucose analyzer (590; Yuwell), while insulin concentration was detected using commercially available RIA kits (cat. no. E-EL-R3034; Elabscience Biotechnology Co., Ltd.).

Histological assessment. The papillary process of liver tissues was obtained and divided into two parts immediately after collection. One part was fixed in a cold buffered $4 \%$ paraformaldehyde solution or cold buffered 3\% glutaraldehyde solution. Following fixation, the first part of tissues in each group was embedded in olefin, cut into 4-mm thick slices and stained with hematoxylin (10 min at room temperature) and eosin ( $2 \mathrm{~min}$ at room temperature) or toluidine blue (3 $\mathrm{min}$ at room temperature) (31). The second part of tissues was treated with Oil Red O (ORO) for $8 \mathrm{~min}$ at room temperature to assess the degree of steatosis (32), and for intraoperative assessment of the steatotic extent to determine whether it can be used as a donor. The degree of steatosis was estimated based on 


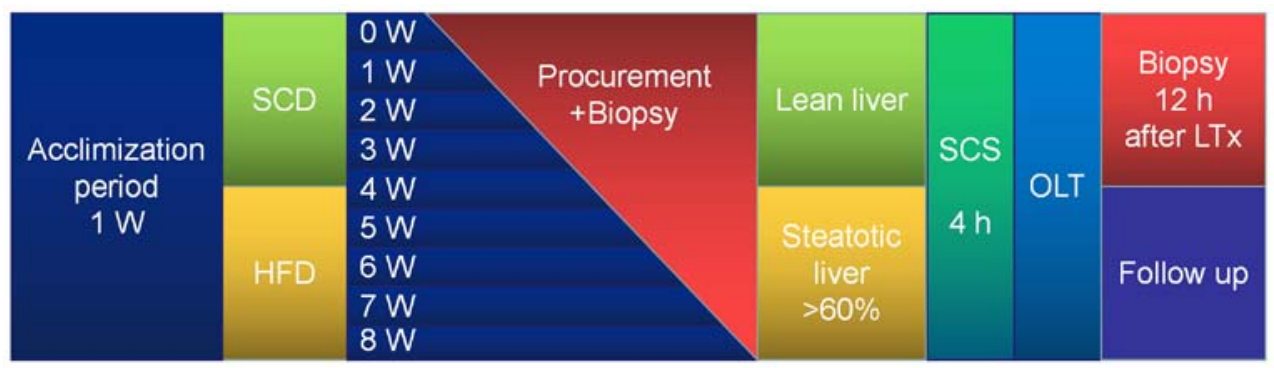

Figure 1. Time schedule of diet-induced period and orthotopic liver transplantation. SCD, standard chow diet; HFD, high-fat diet; W, week; SCS, static cold storage; h, hour; OLT, orthotopic liver transplantation; LTx, liver transplantation.

A

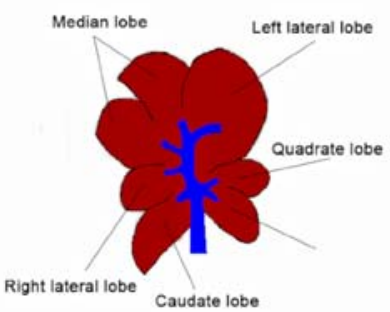

$\mathrm{F}$

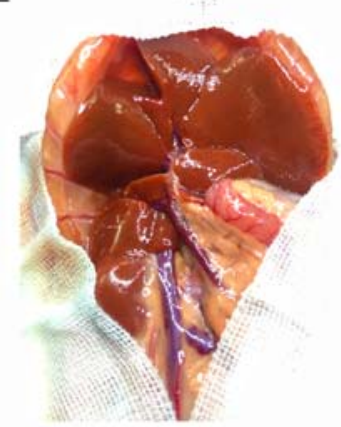

I

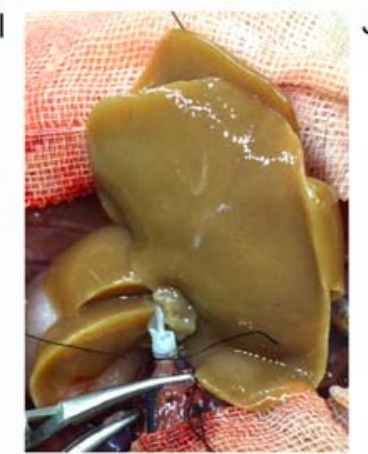

B

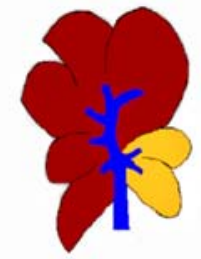

D

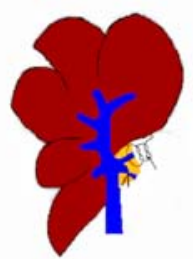

G
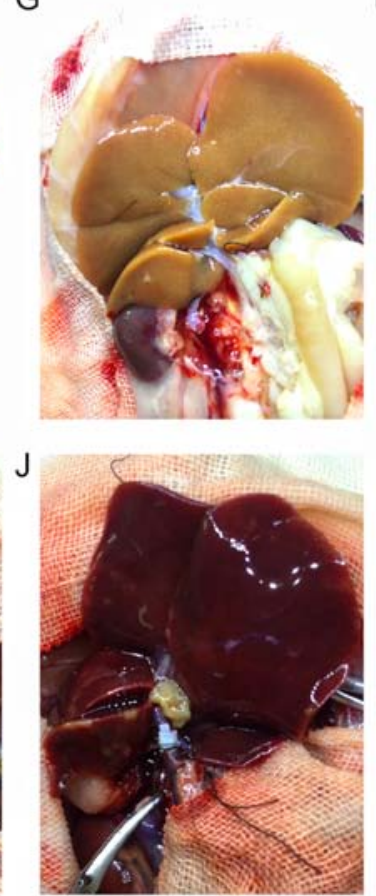

C
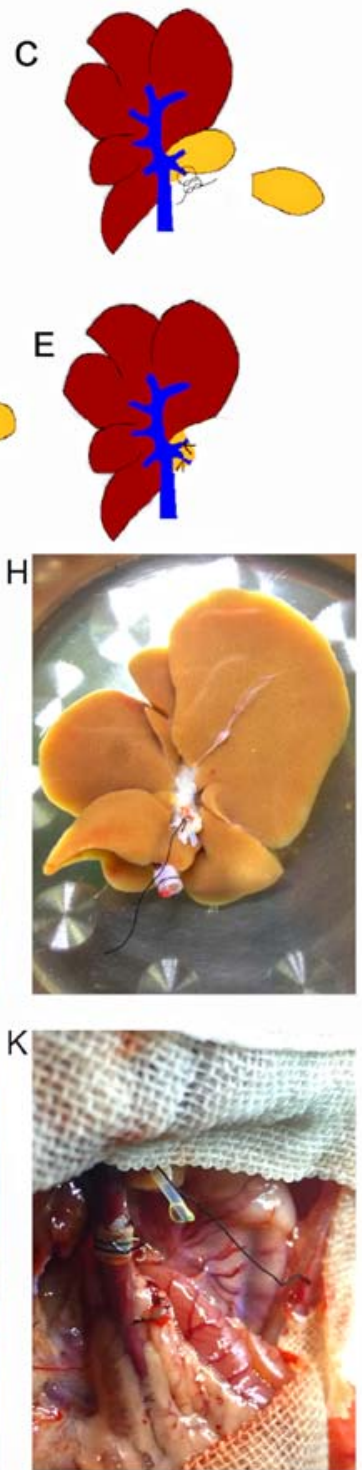

Figure 2. Reduced size procedure prior to transplantation. (A) Schematic representation of hepatic lobes in rats. (B) Two lobes were cut from the whole liver. (C) The papillary process was immediately removed following graft procurement. (D) The quadrate lobe was procured after 4 h of cold storage ex vivo. (E) The remaining five lobes were prepared for transplantation. Liver transplantation process, including $(\mathrm{F})$ pre- and $(\mathrm{G})$ post- hypothermic perfusion, $(\mathrm{H})$ ex vivo preparation, (I) pre- and (J) post- portal vein reperfusion. (K) Bile outflow after reperfusion.

the percentage of hepatocytes containing lipid droplets using Scoring System Definitions (33) and the following formula: Degree of steatotic change $(\%)=[$ (number of hepatocytes with fatty droplets in the all microscopic field)/(number of total hepatocytes in the all microscopic field)] x100. The degree of steatotic change in all groups was determined by calculating the percentage in 10 random areas. A total of two pathologists (Zhongnan Hospital of Wuhan University), blindly assessed and confirmed all biopsies. All liver sections were observed under a light microscope (magnification, x200; TE2000-U; 
Nikon Corporation) and the number and area of fat droplets in hepatocytes were assessed using Image-Pro Plus (version 6.0; Media Cybernetics Inc.).

Orthotopic liver transplantation (OLT). Rats in the HFD group ( $\mathrm{n}=15 ; 6-8$ weeks), with hepatic macrovesicular steatosis $>60 \%$, were subjected to intraoperative ORO staining. OLT was performed as previously described by Kamada and Calne (28). Steatotic liver grafts or lean liver grafts were procured and transplanted into healthy adult rat recipients $4 \mathrm{~h}$ after preservation. For transplantation, the liver of the recipient was removed after clamping the suprahepatic inferior vena cava (SHVC), PV and the IHVC, and grafts were transplanted by anastomosing the SHVC with 8-0 monofilament nylon suture. The cuff was subsequently inserted into the corresponding vessels and secured with 6-0 sutures. The bile duct was anastomosed using an intraluminal stent. The macroscopic changes of liver grafts after portal vein opening were recorded in different groups. A total of five rats in each group were transplanted and observed for 180 days, whereby the survival rate was calculated. For the control group, five rats from the SCD group underwent the same treatment. The transplanted rats were recovered at the Intensive Care Unit Cage (Vetario S10; Brinsea Products Ltd.) and subcutaneously injected with $0.1 \mathrm{mg} / \mathrm{kg}$ buprenorphine (Shanghai Hengyuan Biotechnology Co., Ltd.) twice a day for 3 continuous days post-surgery to relieve pain. To fulfill ethical obligations set by the Swiss legislation, all rats were appropriately evaluated using an animal suffering score, as previously described (34). All transplanted rats were individually housed at a temperature of $23 \pm 1^{\circ} \mathrm{C}$, relative humidity of $55 \pm 10 \%$, air change 12-14 times/h, light/dark cycles of 12/12 h, and had ad libitum access to food and tap water $6 \mathrm{~h}$ post-surgery. The steatotic liver graft was procured from the recipient for biopsy 1-year after LTx.

Statistical analysis. Statistical analysis was performed using GraphPad Prism software (version 4.0; GraphPad Software, Inc.), the non-parametric Mann-Whitney-Wilcoxon test or two-way analysis of variance, followed by Bonferroni test for selected pairs of columns. All data are presented as the mean \pm standard deviation. Survival analysis was performed using the Kaplan Meier method and log-rank test. $\mathrm{P}<0.05$ was considered to indicate a statistically significant difference.

\section{Results}

Body weight. The changes in body weight are presented in Fig. 3A. In weeks 2 and 3, lower body weight was observed in the HFD group compared with the SCD group $(340.70 \pm 16.50$, $310.20 \pm 18.24$ g vs. $375.4 \pm 22.2,388.8 \pm 26.7 \mathrm{~g}$, respectively). However, no significant differences were observed between the groups $(\mathrm{P}>0.05)$.

HFD induces rats to develop dyslipidemia and causes no inflammation. The results of the present study demonstrated changes in ALT and AST expression in rats fed a HFD compared with those fed a SCD; however, no statistical differences were observed between the groups $(\mathrm{P}>0.05$; Fig. 3C and D).
In addition, the level of plasma TG in the HFD group increased by 1.76 -fold at week 2 , and progressively decreased to baseline levels by week 8 . Significantly higher levels of TG were observed in the HFD group compared with the SCD group by week $2(\mathrm{P}<0.05 ; 1.548 \pm 0.172 \mathrm{mmol} / \mathrm{l}$ vs. $0.691 \pm 0.153 \mathrm{mmol} / \mathrm{l})$. The peak TG level was observed at week 4 in the SCD group (Fig. 4). Notably, the levels of plasma TG in both the HFD and $\mathrm{SCD}$ groups were not time-dependent ( $\mathrm{P}>0.05$; Fig. 4A).

The HFD group exhibited small but significantly higher plasma glucose values $(\mathrm{P}<0.05$; Fig. $3 \mathrm{~B})$, accompanied by significantly higher overall plasma insulin values in portal blood ( $\mathrm{P}<0.05$; Fig. 3E) but not in peripheral blood (Fig. 3F), compared with the SCD group. Furthermore, the column demonstrated that liver TG content ascended with time, with a statistically significant difference between the two groups after week 2 ( $\mathrm{P}<0.05$; Fig. 4B).

Morphological assessment. The rat liver receiving HFD was marginally obtuse and became dark yellow in color compared with the SCD-fed rats. In addition, a greasy surface was observed in fatty livers (Fig. 5). Histological analysis demonstrated that the HFD group developed macrovesicular steatosis after 4 weeks of receiving HFD (Fig. 6). The hepatic steatosis aggravated $\sim 10 \%$ per week during the experimental process (Fig. 7).

These results were validated via ORO staining and quantification of TGs. The results demonstrated that intracytoplasmic accumulation of TGs aggravated with time (Fig. 8). The cytoplasm was primarily occupied by several microvesicular steatosis and small fat vacuoles around the nucleus in week 3. However, macrovesicular steatosis was observed in most of the sections in week 6 . The size of the vacuoles increased, pushing the nucleus to the periphery of the cell (Fig. 8).

Post-transplantation graft function and rat survival. Steatotic LTx displayed poor hepatic morphology following reperfusion compared with the normal graft transplantation (Video S1). The reperfusion condition was better in the SCD group compared with the HFD group (Video S1). Furthermore, the liver enzymes were significantly elevated $24 \mathrm{~h}$ after LTx in the HFD group compared with the SCD group (Fig. 9A). The early post-transplantation survival rates on day 7,30 and 365 were $80 \%(4 / 5), 20 \%(1 / 5)$ and $20 \%(1 / 5)$ in the HFD group, and $100 \%(5 / 5), 100 \%(5 / 5)$ and $100 \%(5 / 5)$ in the SCD group (Fig. 9B). Among the rats that accepted severely steatotic grafts, only one rat survived, while the other four died 8 days post-surgery ( $8 \pm 2$ days). The postmortem examination revealed massive ascites, and it was concluded that the rats died from PNF (4/5). Macrovesicular steatosis dissipated from severely steatotic liver graft 1-year after LTx (Fig. 10).

\section{Discussion}

Currently, several fatty liver animal models have been developed, including rats, mice, the sand rat, rabbit, duck, geese and miniature swine (35-40). The rat model, which is the most commonly used fatty liver animal model, can be classified into three types: Congenital, genetically modified and food/drug-induced model (35-40). However, the former two 
A
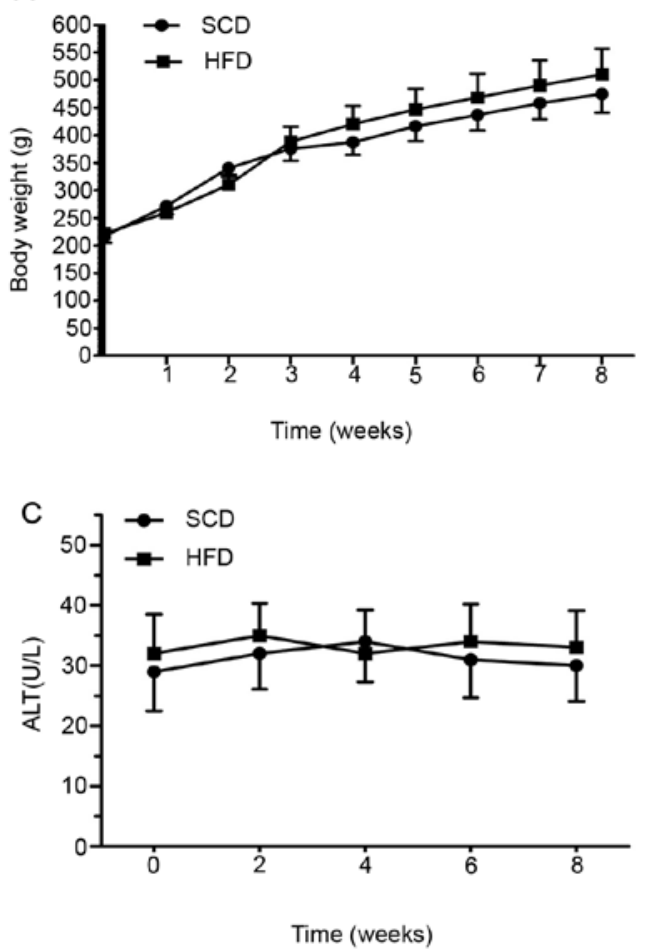

$E$

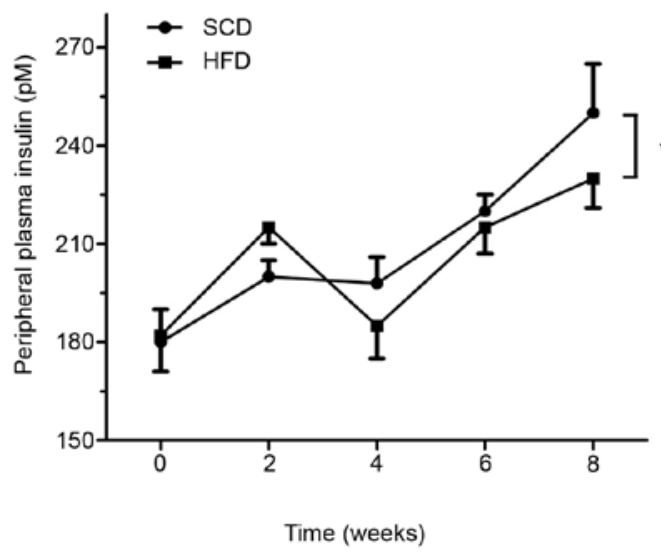

B
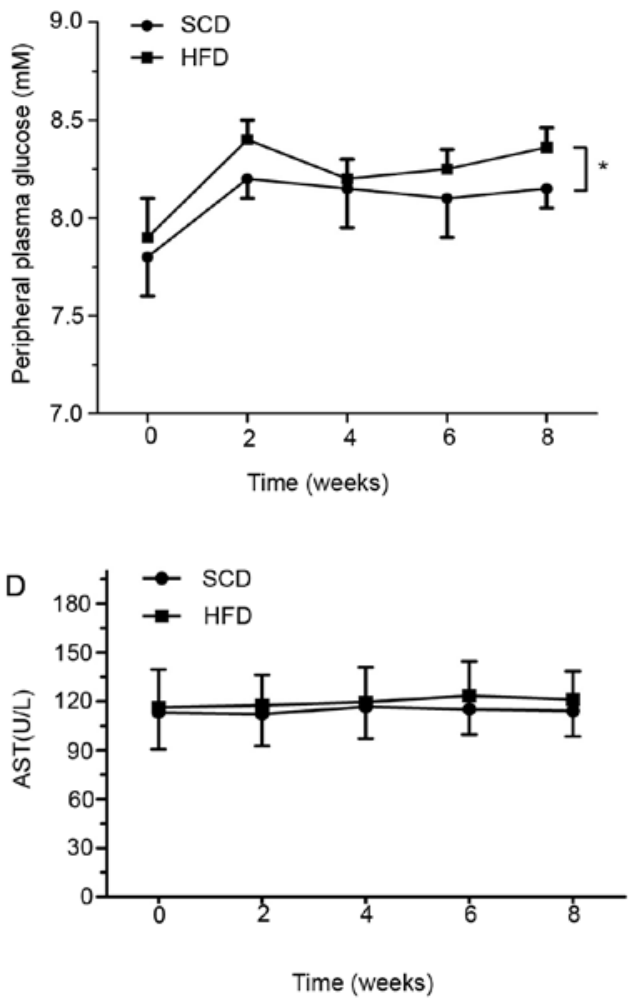

$\mathrm{F}$

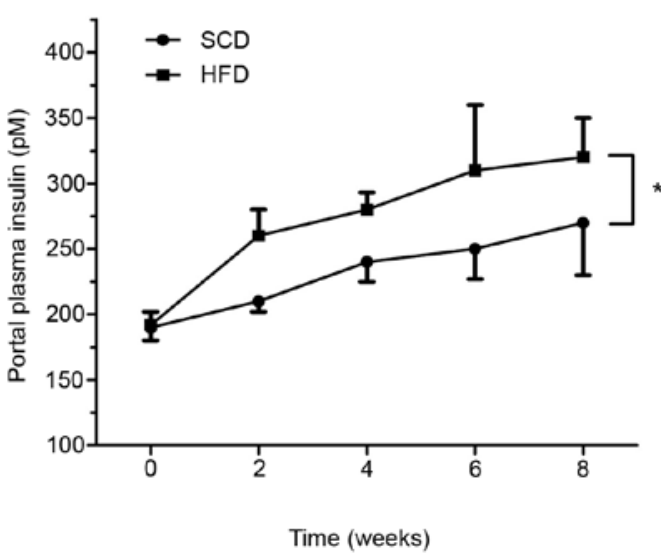

Figure 3. Body weight and serum levels in HFD and SCD groups. (A) The changes in body weight. Plasma (B) glucose, (C) ALT and (D) AST levels. Plasma insulin concentrations were measured in (E) peripheral and (F) portal blood. $n=5$ and data are presented as the mean \pm standard deviation. ${ }^{*} \mathrm{P}<0.05$. HFD, high-fat diet; SCD, standard chow diet; ALT, alanine aminotransaminase; AST, aspartate aminotransferase.

are not in conformity with the pathogenesis of the human disease. In the present study, food-induction was used to induce hepatic steatosis and simultaneously exclude other interference factors (41). Volume reduction was implemented as a self-control method, making the data in the control group more accurate without enlarging samples, which is in line with the 3Rs concept of animal experiments (replacement, reduction and refinement) (42). OLT was performed using normal grafts, with or without a $20 \%$ volume reduction to verify the safety of this method. Post-surgery survival rates were assessed within the two groups (100 vs. 100\%).

Regarding body weight, lower body weight was observed in the HFD group compared with the SCD group; however, no significant differences were observed between the two groups. The lower body weight may be attributed to a time period of adaptation for HFD rats to their diet. In addition, the diameter of PV, SHVC, IHVC and bile duct was narrower in rats fed with HFD compared with those fed with SCD.

The pathogenesis of fatty liver remains unclear; however, it is speculated that its formation is associated with fat metabolism disorders, increase in fat synthesis and oxidation-reduction, as well as the imbalance in synthesis or discharge of TG and LDL (12). The results of the present study demonstrated no significant differences in ALT and AST levels between the HFD and SCD groups, which indicated the absence of additional steatotic liver injury. In addition, the enzymes did not increase with the aggravation of hepatic steatosis.

Notably, TC was not consistent with the degree of hepatic steatosis, as speculated. Conversely, no linear association was observed between TG levels in serum and liver. As an integral part of the metabolic syndrome, hepatic steatosis is associated with the development of insulin resistance $(43,44)$. In the 

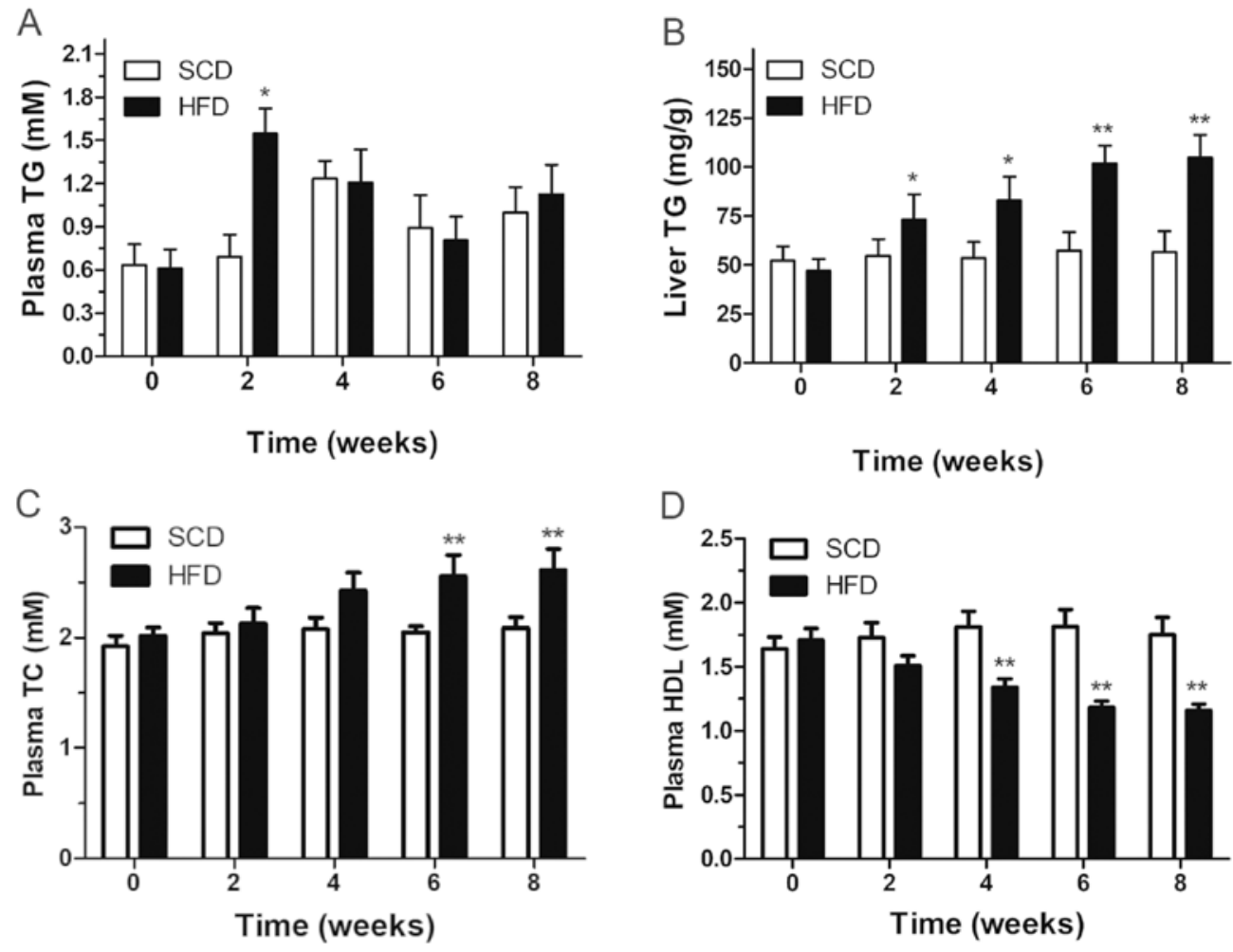

Time (weeks)
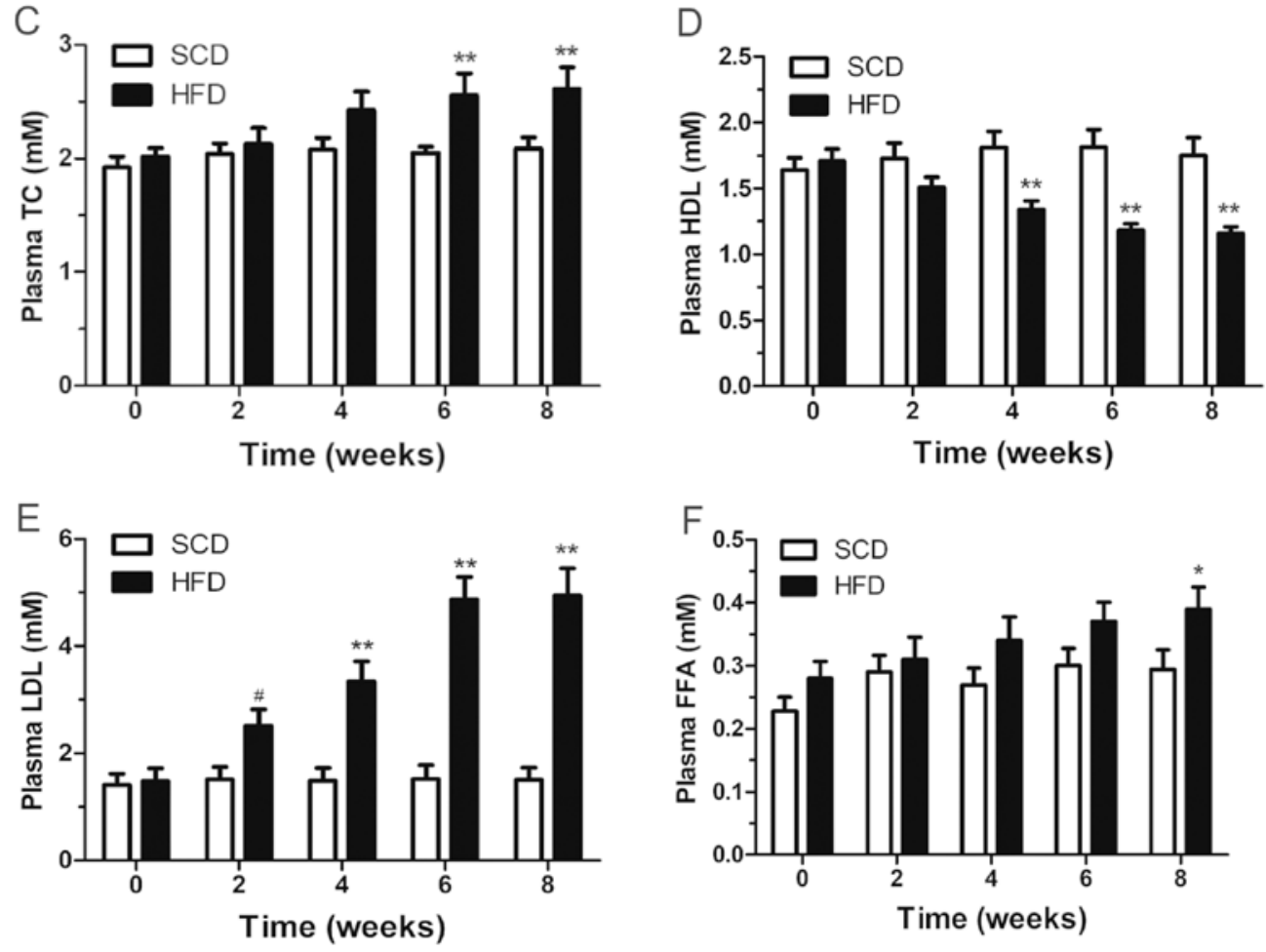

Figure 4. Plasma (A) TG, (B) liver TG, (C) plasma TC, (D) HDL, (E) LDL and (F) FFA levels in rats fed a HFD or SCD. Date are presented as the mean \pm standard deviation. SCD compared with HFD, "P<0.05 and ${ }^{* *} \mathrm{P}<0.01$ vs. SCD. TG, triglyceride; TC, cholesterol; HDL, high density lipoprotein; LDL, low density lipoprotein; FFA, free fatty acid; HFD, high-fat diet; SCD, standard chow diet.
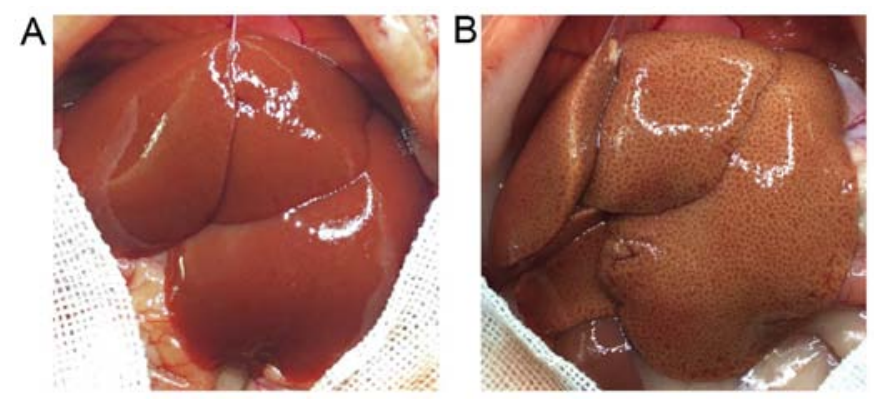

Figure 5. General observations of (A) normal liver and (B) steatotic liver (B) in situ.

present study, the HFD group was significantly associated with higher plasma glucose levels and higher portal insulin levels. These higher portal insulin levels in HFD rats may participate in the rapid development of hepatic steatosis. This may be one reason as to why some steatotic grafts reach full recovery following implantation in recipients with no insulin resistance.

In the present study, the steatotic liver was larger in size, has a fatty surface, obtuse margin and was a dark yellow color compared with the healthy liver (SCD group). In addition, no signs of fibrosis were observed in any of the groups. Histopathological analyses demonstrated that the hepatocytes of HFD rats had little lipid droplets 1 week after the model was established, and the number of hepatocytes increased in a time-dependent manner. After 2 weeks, the little lipid drops demonstrated a mutual-fusion-trend, indicating microvesicular steatosis. A portion of microvesicular lipid droplets started to fuse into macrovesicular fat vacuoles at week 3 , and notable macrovesicular steatosis was observed at week 4, which progressively decreased up to week 6 . In addition, fat vacuoles were observed in the hepatocytes, and their nuclei were squeezed to one side, revealing the irregular shape of hepatocytes and hepatic sinusoid constriction, while the field of vision was filled with macro fat vacuoles. The hepatocytes 

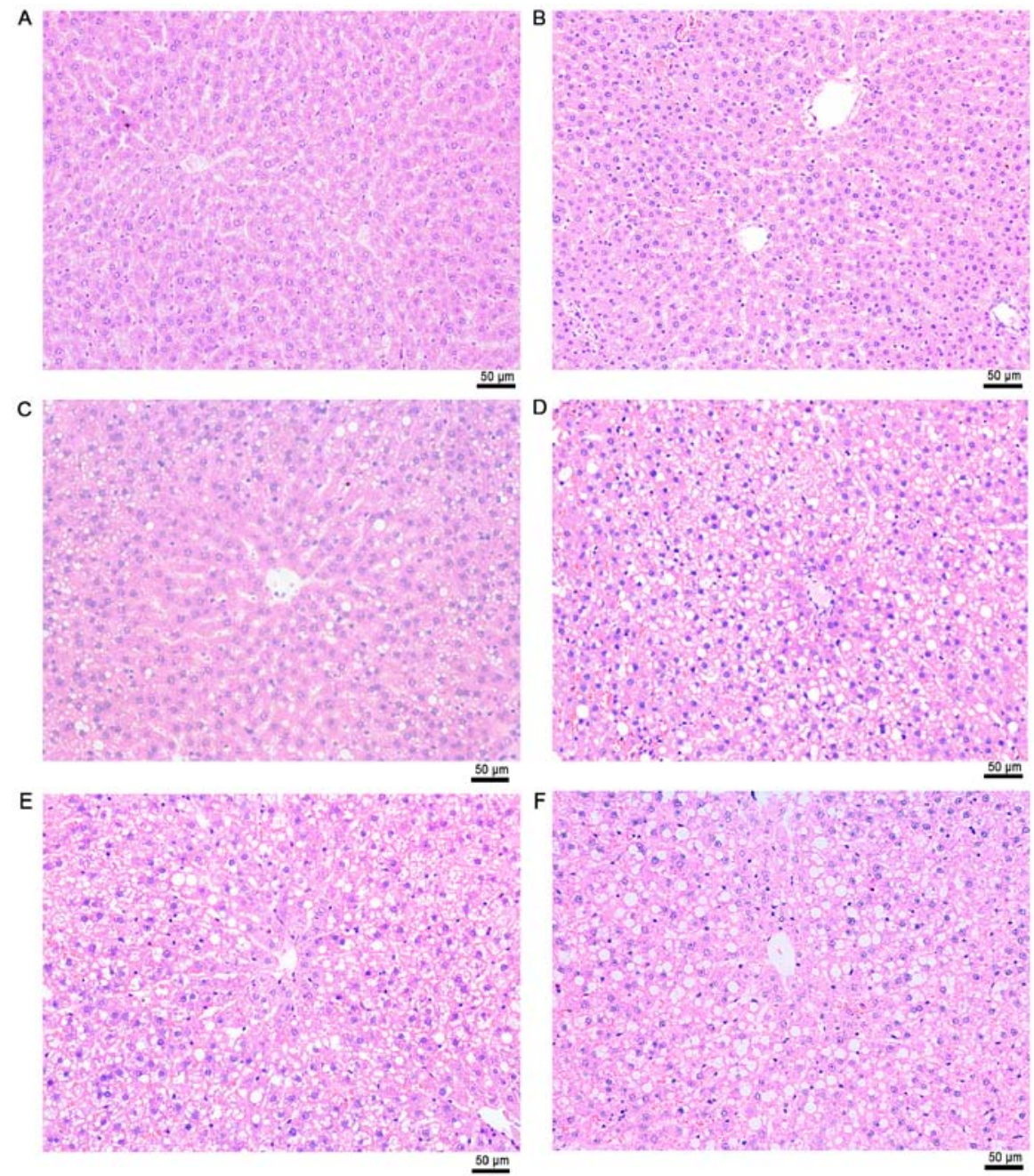

Figure 6. Hematoxylin and eosin stained liver tissues from high-fat diet observed under a light microscope at week (A) 0 , (B) 1 , (C) 2 , (D) 4 , (E) 6 and (F) 8 . Scale bar, $50 \mu \mathrm{m}$. Magnification, x200.

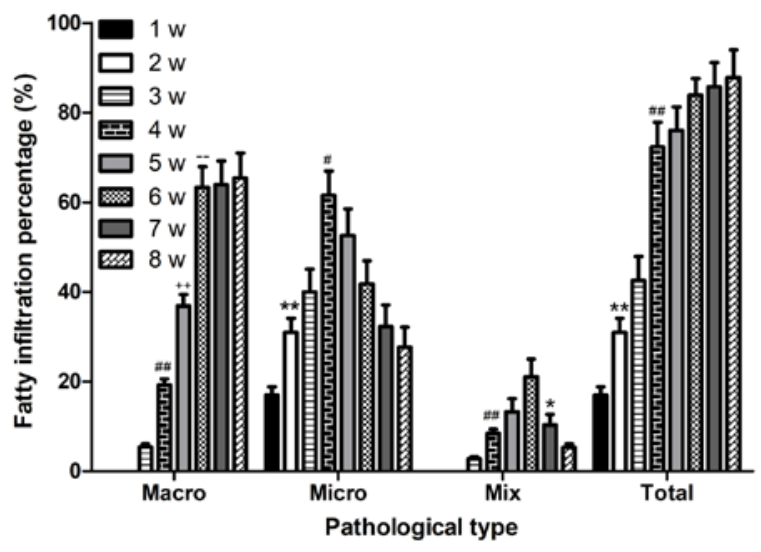

Figure 7. Degree of steatotic change in different groups. Mix refers to hepatocytes in which both macro and micro vacuoles were observed. $n=5$ and data are presented as the mean \pm standard deviation. ${ }^{*} \mathrm{P}<0.05$ and ${ }^{* *} \mathrm{P}<0.01$ vs. $1 \mathrm{w}$; ${ }^{\#} \mathrm{P}<0.05$ and ${ }^{\# \#} \mathrm{P}<0.01$ vs. $3 \mathrm{w} ;{ }^{++} \mathrm{P}<0.01$ vs. $4 \mathrm{w} ;{ }^{-"} \mathrm{P}<0.01$ vs. 5 w. w, week.

in the previous 3 weeks predominantly revealed microvesicular steatosis, while they primarily revealed macrovesicular steatosis in the latter weeks. A more significant difference was observed between images at weeks 3 and 6 via $H \& E$ and ORO staining.
Based on this model, strategies such as, ischemic preconditioning, pharmacological preconditioning and machine perfusion may be used to enhance graft quality $(6,34)$. Although the steatotic liver model was stabilized by two preliminary experiments, the results depended on the energy intake and individual differences. Regrouping would be more accurate based on TG content in tissues, combined with the degree of hepatic steatosis estimated by ORO staining.

LTx was performed using steatotic grafts or normal liver. Clinically, hepatic macrovesicular steatosis $>60 \%$ is considered a contraindication for transplantation due to the high morbidity of PNF (10). With reference to long-term survival, rats receiving SCD survived longer than those fed a HFD; however, no statistically significant differences were observed in short-term survival between the two groups. Restoring the rat intestinal blood flow, relieving congestion and stabilizing the hemodynamics are critical features for improving animal survival (28). In the present study, an hepatic phase was limited between 20-22 min, including the time for suturing the SHVC, cuffing and opening the PV, outflowing and clamping the IHVC, removing the SHVC clamp and letting approximately $0.2 \mathrm{ml}$ blood outflow from the IHVC in case thrombus or other impurities circulated into the blood when opening 


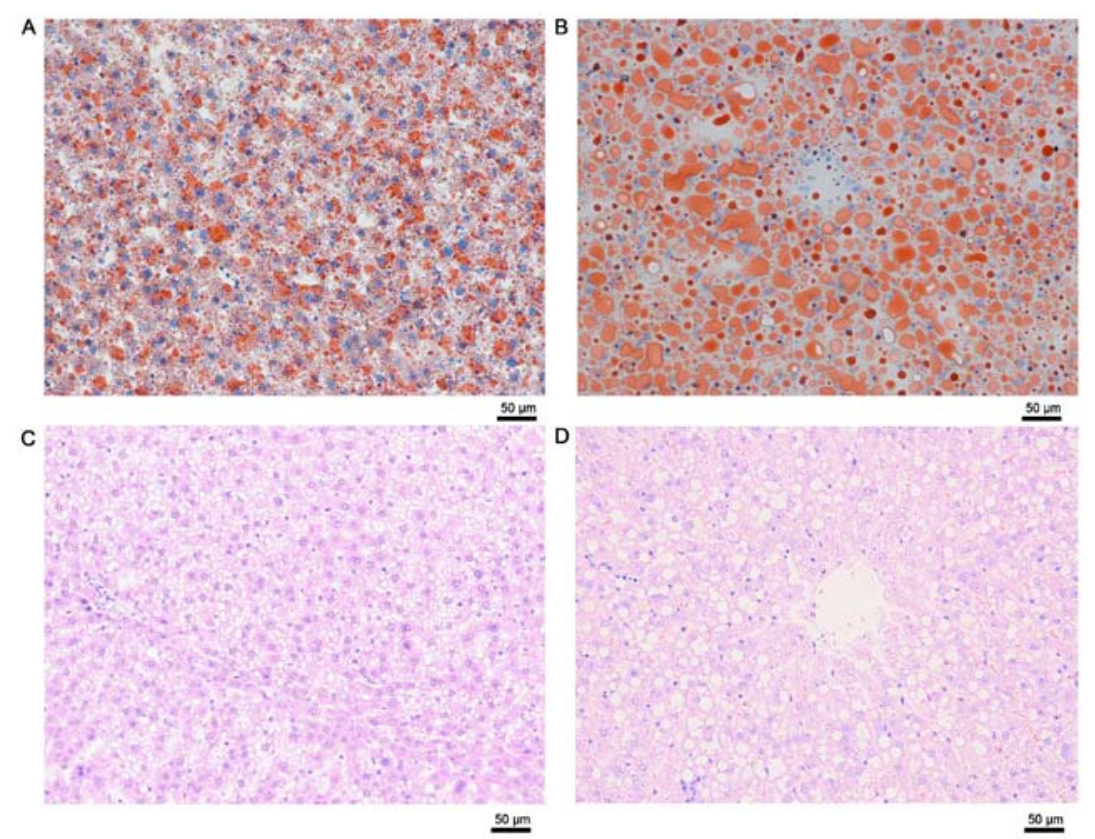

Figure 8. (A and B) Oil Red $\mathrm{O}$, and (C and D) hematoxylin and eosin stained liver tissue sections from the high-fat diet group at weeks (A and C) 3 and (B and D) 6. Microvesicular steatosis was observed at week 3, while macrovesicular steatosis was observed at week 6. Scale bar, $50 \mu \mathrm{m}$. Magnification, x200.
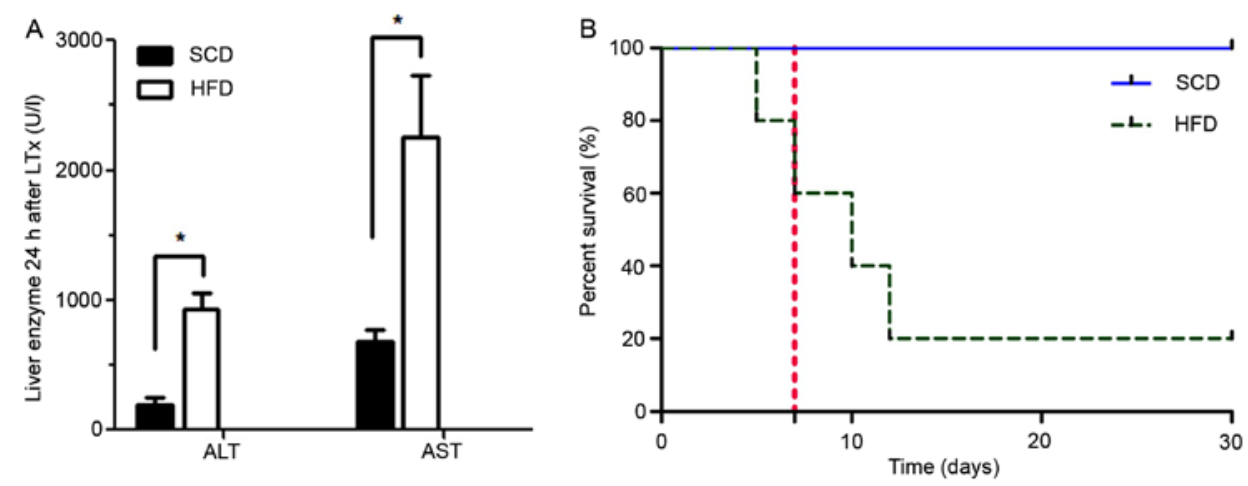

Figure 9. Post-transplantation survival of rats and (A) liver enzymes in the HFD and SCD groups. (B) The 1- and 4-week survival rates were 80\% (4/5) and $20 \%$ (1/5) for recipients of grafts from steatotic liver, whereas the survival rates were $100 \%(5 / 5)$ and $100 \%(5 / 5)$ for recipients of grafts from normal liver, respectively. $\mathrm{n}=5$ and data are presented as the mean \pm standard deviation. ${ }^{*} \mathrm{P}<0.05$. HFD, high-fat diet; SCD, standard chow diet; LTx, liver transplantation; $\mathrm{h}$, hour; ALT, alanine aminotransaminase; AST, aspartate aminotransferase.
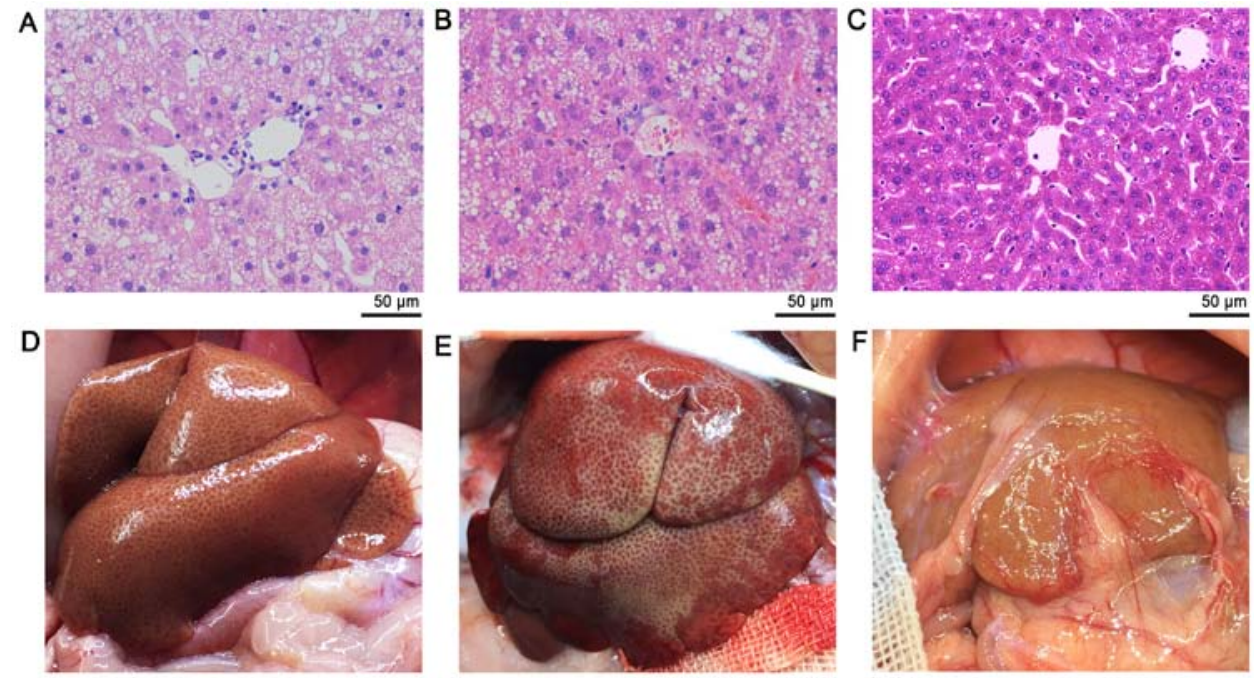

Figure 10. Steatotic liver graft changes pre-, during and post-transplantation. Macroscopy and microscopy of the steatotic liver graft (A and D) prior to procurement, (B and E) during transplantation and (C and F) 1 year after transplantation. Scale bar, $50 \mu \mathrm{m}$. Magnification, x 200. 
PV. The results of the present study demonstrated that fatty LTx displayed poor hepatic morphology following PV reperfusion compared with the normal graft transplantation. All of the steatotic grafts appeared to be male-reperfusion during the transplantation. The liver surface appeared to be piebald due to the uneven blood reperfusion. In addition, combined with the post-surgery survival, the intraoperative reperfusion situation of the liver did not have a decisive role in predicting rat prognosis, it was not possible to determine whether the rats would achieve a good outcome just based on the reperfusion morphology. In one case of fatty LTx in the present study, nearly no blood reperfusion was observed on the liver surface except in the portal vein and its branches. However, despite the poor performance, this was the only case that survived more than 6 months, longer than the others that had even better reperfusion. As a result, the prognosis could not be predicted only by intraoperative morphology in steatotic LT.

The steatotic liver is vulnerable to ischemic injury (21). PNF was commonly observed in the severely steatotic liver group that had sustained $4 \mathrm{~h} \mathrm{CS}$. The post-surgery rats only survived if the liver function was restored, usually within 2 weeks. In the present study, except for one rat in the HFD group that survived, four rats died $8 \pm 2$ days after the surgery. In total, $80 \%$ of rats did not recover from PNF until their death. As steatotic liver leads to poor prognosis, the authors aim to use machine perfusion in future studies to improve the survival outcomes.

In conclusion, the results of the present study suggest that HFD may be used to induce stable and rapid hepatic steatosis in rats without inducing inflammation. In addition, the lipid droplets accumulate into fat vacuoles over time, from microvesicular steatosis into macrovesicular steatosis. Furthermore, volume reduction, as a self-control method, may be applied in steatotic LTx without increasing the number of animals being used, thus promoting animal welfare. However, therapeutic approaches or machine perfusion need to be implemented in steatotic grafts to improve the quality of donated organs and improve the post-transplantation survival.

\section{Acknowledgements}

Not applicable.

\section{Funding}

The present study was funded by the National Natural Science Foundation of China, (grant no. 81970548), the Medical Science Advancement Program (Youth Scholars) of Wuhan University (grant no. TFZZ2018035) and the Zhongnan Hospital of Wuhan University Science, Technology and Innovation Seed Fund (grant no. ZNPY2018010).

\section{Availability of data and materials}

The datasets used and/or analyzed during the current study are available from the corresponding author on reasonable request.

\section{Authors' contributions}

LF, ZF and QY contributed to the conception and design. LF, ZF, YX and SY performed the experiments. YX, SY, YW and
GP contributed to data acquisition and analysis. All authors have read and approved the final manuscript.

\section{Ethics approval and consent to participate}

The present study was approved (IRB approval no. AF-177) by Wuhan University Institutional Animal Care and Use Committee (Wuhan, China) and all animal experiments were performed in accordance with the Association for the Assessment and Accreditation of Laboratory Animal Care and Institutional Animal Care and Use Committee regulations and guidelines.

\section{Patient consent for publication}

Not applicable.

\section{Competing interests}

The authors declare that they have no competing interests.

\section{References}

1. Starzl TE, Marchioro TL, Von Kaulla KN, Hermann G, Brittain RS and Waddell WR: Homotransplantatin of the liver in humans. Surg Gynecol Obstet 117: 659-676, 1963.

2. Wolfe RA, Merion RM, Roys EC and Port FK: Trends in organ donation and transplantation in the United States, 1998-2007. Am J Transplant 9: 869-878, 2009.

3. Wertheim JA, Petrowsky H, Saab S, Kupiec-Weglinski JW and Busuttil RW: Major challenges limiting liver transplantation in the United States. Am J Transplant 11: 1773-1784, 2011.

4. Durand F, Renz JF, Alkofer B, Burra P, Clavien PA, Porte RJ, Freeman RB and Belghiti J: Report of the Paris consensus meeting on expanded criteria donors in liver transplantation. Liver Transpl 14: 1694-1707, 2008.

5. Berg CL, Steffick DE, Edwards EB, Edwards EB, Heimbach JK, Magee JC, Washburn WK and Mazariegos GV: Liver and intestine transplantation in the United States 1998-2007. Am J Transplant 9: 907-931, 2009.

6. Henry SD and Guarrera JV: Protective effects of hypothermic ex vivo perfusion on ischemia/reperfusion injury and transplant outcomes. Transplant Rev (Orlando) 26: 163-175, 2012.

7. Briceño J, Marchal T, Padillo J, Solórzano G and Pera C: Influence of marginal donors on liver preservation injury. Transplantation 74: 522-526, 2002.

8. Salizzoni M, Franchello A, Zamboni F, Ricchiuti A, Cocchis D, Fop F, Brunati A and Cerutti E: Marginal grafts: Finding the correct treatment for fatty livers. Transpl Int 16: 486-493, 2003.

9. Tekin K, Imber CJ, Atli M, Gunson BK, Bramhall SR, Mayer D, Buckels JA, McMaster P and Mirza DF: A simple scoring system to evaluate the effects of cold ischemia on marginal liver donors. Transplantation 77: 411-416, 2004.

10. Cameron A and Busuttil RW: AASLD/ILTS transplant course: Is there an extended donor suitable for everyone? Liver Transpl 11 (suppl 2): S2-S5, 2005

11. Shaker M, Tabbaa A, Albeldawi M and Alkhouri N: Liver transplantation for nonalcoholic fatty liver disease: New challenges and new opportunities. World J Gastroenterol 20: 5320-5330, 2014.

12. Rinella ME: Nonalcoholic fatty liver disease: A systematic review. JAMA 313: 2263-2273, 2015.

13. Browning JD, Szczepaniak LS, Dobbins R, Nuremberg P, Horton JD, Cohen JC, Grundy SM and Hobbs HH: Prevalence of hepatic steatosis in an urban population in the United States: Impact of ethnicity. Hepatology 40: 1387-1395, 2004.

14. Neuschwander-Tetri BA and Caldwell SH: Nonalcoholic steatohepatitis: Summary of an AASLD Single topic conference. Hepatology 37: 1202-1219, 2003.

15. Ruhl CE and Everhart JE: Fatty liver indices in the multiethnic united states national health and nutrition examination survey. Aliment Pharmacol Ther 41: 65-76, 2015. 
16. Sanyal AJ; American Gastroenterological Association: AGA technical review on nonalcoholic fatty liver disease. Gastroenterology 123: 1705-1725, 2002.

17. McCormack L, Dutkowski P, El-Badry AM and Clavien PA: Liver transplantation using fatty livers: Always feasible? J Hepatol 54: 1055-1062, 2011.

18. Ploeg RJ, D'Alessandro AM, Knechtle SJ, Stegall MD, Pirsch JD, Hoffmann RM, Sasaki T, Sollinger HW, Belzer FO and Kalayoglu M: Risk factors for primary dysfunction after liver transplantation-a multivariate analysis. Transplantation 55 807-813, 1993.

19. Busuttil RW and Tanaka K: The utility of marginal donors in liver transplantation. Liver Transpl 9: 651-663, 2003.

20. Selzner M and Clavien PA: Fatty liver in liver transplantation and surgery. Semin Liver Dis 21: 105-113, 2001.

21. Sun CK, Zhang XY,Zimmermann A, Davis G and Wheatley AM: Effect of ischemia-reperfusion injury on the microcirculation of the steatotic liver of the Zucker rat. Transplantation 72: 1625-1631, 2001

22. Imber CJ, St Peter SD, Lopez I, Guiver L and Friend PJ: Current practice regarding the use of fatty livers: A trans- Atlantic survey. Liver Transpl 8: 545-549, 2002.

23. El-Badry AM, Moritz W, Contaldo C, Tian Y, Graf R and Clavien PA: Prevention of reperfusion injury and microcirculatory failure in macrosteatotic mouse liver by omega-3 fatty acids. Hepatology 45: 855-863, 2007.

24. Todo S, Demetris AJ, Makowka L, Teperman L, Podesta L, Shaver T, Tzakis A and Starzl TE: Primary nonfunction of hepatic allografts with preexisting fatty infiltration. Transplantation 47 903-905, 1989.

25. Verran D, Kusyk T, Painter D, Fisher J, Koorey D, Strasser S Stewart G and McCaughan G: Clinical experience gained from the use of 120 steatotic donor livers for orthotopic liver transplantation. Liver Transpl 9: 500-505, 2003.

26. Gabrielli M, Moisan F, Vidal M, Duarte I, Jiménez M, Izquierdo G, Domínguez P, Méndez J, Soza A, Benitez C, et al: Steatotic livers. Can we use them in OLTX? Outcome data from a prospective baseline liver biopsy study. Ann Hepatol 11: 891-898, 2012.

27. Spitzer AL, Lao OB, Dick AA, Bakthavatsalam R, Halldorson JB, Yeh MM, Upton MP, Reyes JD and Perkins JD: The biopsied donor liver: Incorporating macrosteatosis into high-risk donor assessment. Liver Transpl 16: 874-884, 2010.

28. Kamada N and Calne RY: Orthotopic liver transplantation in the rat. Technique using cuff for portal vein anastomosis and biliary drainage. Transplantation 28: 47-50, 1979.

29. Hijona E, Hijona L, Arenas JI and Bujanda L: Inflammatory mediators of hepatic steatosis. Mediators Inflamm 2010: 837419, 2010.

30. Bligh EG and Dyer WJ: A rapid method of total lipid extraction and purification. Can J Biochem Physiol 37: 911-917, 1959.

31. Lattouf R, Younes R, Lutomski D, Naaman N, Godeau G, Senni K and Changotade S: Picrosirius red staining: A useful tool to appraise collagen networks in normal and pathological tissues. J Histochem Cytochem 62: 751-758, 2014.
32. Mehlem A, Hagberg CE, Muhl L, Eriksson U and Falkevall A: Imaging of neutral lipids by oil red $\mathrm{O}$ for analyzing the metabolic status in health and disease. Nat Protoc 8: 1149-1154, 2013.

33. Kleiner DE, Brunt EM, Van Natta M, Behling C, Contos MJ, Cummings OW, Ferrell LD, Liu YC, Torbenson MS, Unalp-Arida A, et al: Design and validation of a histological scoring system for nonalcoholic fatty liver disease. Hepatology 41 : 1313-1321, 2005

34. de Rougemont O, Breitenstein S, Leskosek B, Weber A, Graf R, Clavien PA and Dutkowski P: One hour hypothermic oxygenated perfusion (HOPE) protects nonviable liver allografts donated after cardiac death. Ann Surg 250: 674-683, 2009.

35. Corominas J, Marchesi JA, Puig-Oliveras A, Revilla M, Estellé J, Alves E, Folch JM and Ballester M: Epigenetic regulation of the ELOVL6 gene is associated with a major QTL effect on fatty acid composition in pigs. Genet Sel Evol 47: 20, 2015.

36. Tuvdendorj D, Zhang XJ, Chinkes DL, Wang L, Wu Z, Rodriguez NA, Herndon DN and Wolfe RR: Triglycerides produced in the livers of fasting rabbits are predominantly stored as opposed to secreted into the plasma. Metabolism 64: 580-587, 2015.

37. Katz DL: Ducks, geese, faith, and fatty livers. Child Obes 10: 373-374, 2014.

38. Awde S, Marty-Gasset N, Prahkarnkaeo K and Rémignon H: Relationship between proteolytic activities and cooking loss variability in liver issued from force-fed mule ducks. J Agric Food Chem 62: 3262-3268, 2014.

39. Spolding B, Connor T, Wittmer C, Abreu LL, Kaspi A, Ziemann M, Kaur G, Cooper A, Morrison S, Lee S, et al: Rapid development of non-alcoholic steatohepatitis in Psammomys obesus (Israeli sand rat). PLoS One 9: e92656, 2014.

40. Gauthier MS, Favier R and Lavoie JM: Time course of the development of non-alcoholic hepatic steatosis in response to high-fat diet-induced obesity in rats. Br J Nutr 95: 273-281, 2006.

41. Matteoni CA, Younossi ZM, Gramlieh T, Boparai N, Liu YC and McCullough AJ: Nonalcoholic fatty liver disease: A spectrum of clinical and pathological severity. Gastroenterology 116: 1413-1419, 1999.

42. Flecknell P: Replacement, reduction and refinement. ALTEX 19: 73-78, 2002.

43. Saltiel AR and Kahn CR: Insulin signalling and the regulation of glucose and lipid metabolism. Nature 414: 799-806, 2001.

44. Samuel VT, Liu ZX, Qu X, Elder BD, Bilz S, Befroy D, Romanelli AJ and Shulman GI: Mechanism of hepatic insulin resistance in non-alcoholic fatty liver disease. J Biol Chem 279: 32345-32353, 2004.

(i) (9) This work is licensed under a Creative Commons Attribution-NonCommercial-NoDerivatives 4.0 International (CC BY-NC-ND 4.0) License. 